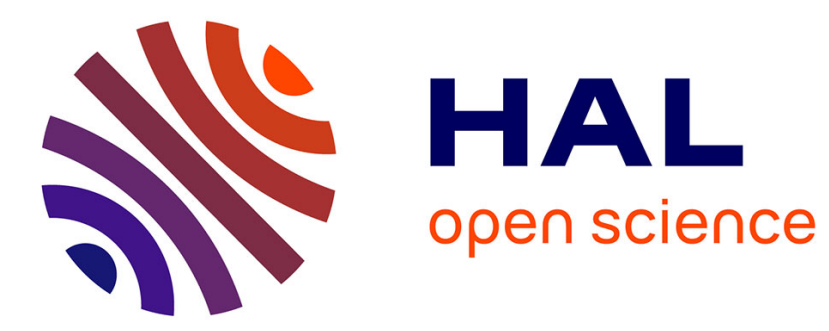

\title{
Arithmetic properties of summands of partitions II
}

Cécile Dartyge, Andras Sarkozy

\section{To cite this version:}

Cécile Dartyge, Andras Sarkozy. Arithmetic properties of summands of partitions II. Ramanujan Journal (The), 2005, 10, pp.383-394. hal-00091844

\section{HAL Id: hal-00091844 https://hal.science/hal-00091844}

Submitted on 7 Sep 2006

HAL is a multi-disciplinary open access archive for the deposit and dissemination of scientific research documents, whether they are published or not. The documents may come from teaching and research institutions in France or abroad, or from public or private research centers.
L'archive ouverte pluridisciplinaire HAL, est destinée au dépôt et à la diffusion de documents scientifiques de niveau recherche, publiés ou non, émanant des établissements d'enseignement et de recherche français ou étrangers, des laboratoires publics ou privés. 
$(4 / 12 / 2003,16 \mathrm{~h} 09)$

\title{
Arithmetic properties of summands of partitions II
}

\author{
Cécile Dartyge and András Sárközy
}

\begin{abstract}
Let $d \in \mathbb{N}, d \geqslant 2$. We prove that a positive proportion of partitions of an integer $n$ satisfies the following : for all $1 \leqslant a<b \leqslant d$, the number of the parts congruent to $a(\bmod d)$ is greater than the number of the parts congruent to $b(\bmod d)$. We also show that for almost all partitions the rate of the number of square free parts is $\frac{6}{\pi^{2}}(1+o(1))$. 1. Introduction
\end{abstract}

Let $n, d \in \mathbb{N}$ and $a \in \mathbb{Z}$ with $2 \leqslant d \leqslant n$. For a partition $\lambda=\left(\lambda_{1}, \ldots, \lambda_{s}\right)$ of $n=\lambda_{1}+\cdots+\lambda_{s}$ with $\lambda_{1} \geqslant \ldots \geqslant \lambda_{s}$ we denote by $s(\lambda)=s$ the number of parts in $\lambda$ and we define

$$
F_{a, d}(\lambda):=\sum_{\substack{1 \leqslant j \leqslant s \\ \lambda_{j} \equiv a(\bmod d)}} 1 .
$$

Let $\mathcal{P}(n)$ be the set of all partitions of $n$. We will also use the standard notation $p(n)$ for the number of partitions of $n$ and we write:

$$
C:=\pi \sqrt{\frac{2}{3}}
$$

In [1] we proved that for almost all partitions the parts are well distributed in residue classes modulo $d$ but we observed that this uniformity is limited by the fact that the small parts occur more frequently. The first part of this article is devoted to the study of this phenomenon. First we will prove a theorem which sharpens Theorem 1.4 of [1]

Theorem 1.1. Let $d \geqslant 2$. There exists $n_{0}=n_{0}(d)$ such that for $n>n_{0}$, $0<a<b \leqslant d$ there are more than $\frac{p(n)}{12}$ partitions $\lambda$ with

$$
F_{a, d}(\lambda)-F_{b, d}(\lambda)>\frac{(a+b) \sqrt{n}}{50 a b}
$$


In [1] we stated the inequality $\left|F_{a, d}(\lambda)-F_{b, d}(\lambda)\right|>\frac{(a+b) \sqrt{n}}{50 a b}$ for at least $p(n) / 6$ partitions, the novelty of Theorem 1.1 is that there is no absolute value in it.

In [1] we defined the notion of " $d$-regular" partitions: these are partitions $\lambda$ with $F_{a, d}(\lambda)>F_{a^{\prime}, d}(\lambda)$ for any $1 \leqslant a<a^{\prime} \leqslant d$. In the first step of the study of these partitions we will prove that for a positive proportion of partitions the parts congruent to $\equiv 1(\bmod d)$ are more frequent than the others :

Theorem 1.2. For $d \geqslant 2$, there are more than $p(n) / d$ partitions $\lambda$ of $n$ such that $F_{1, d}(\lambda) \geqslant F_{a, d}(\lambda)$ for all $2 \leqslant a \leqslant d$.

With combinatorics arguments we will also prove that there are "many" $d$-regular partitions:

Theorem 1.3. For $d \geqslant 2$ there are more than $\frac{p(n)}{d !}\left(1+O\left(\frac{d ! d^{4}}{\sqrt{n}}\right)\right) d$-regular partitions.

The second part of this paper is devoted to the study of the number of the square free parts. We will prove that for almost all partitions this number is as expected:

Theorem 1.4. For almost all partitions $\lambda$ of $n$ the number of the square free parts is $s(\lambda) \frac{6}{\pi^{2}}(1+o(1))$.

When we want to prove that an arbitrary finite sequence $\mathcal{A}$ of integers contains many square free integers, we usually need for large $d$ an upper bound for the number of integers $n \in \mathcal{A}$ such that $d \mid n$.

Here Proposition 1.5 will play this role:

Proposition 1.5. For any $8 \leqslant d \leqslant n$, there are at most $\frac{p(n)}{d^{2}}$ partitions $\lambda$ of $n$ such that

$$
F_{0, d}(\lambda) \geqslant 100 \frac{\sqrt{n} \log n \log ^{2} d}{d}
$$

With more work our results could be sharpened slightly. In fact, we will see that a direct consequence of a lemma of Szalay and Turán [5] is that for at most $\frac{p(n)}{n^{2}}$ partitions $\lambda$ there exists a $d>\frac{5 \sqrt{6}}{2 \pi} \sqrt{n} \log n$ such that $F_{0, d}(\lambda)>0$. 


\section{Proof of Theorem 1.1}

Let $A$ and $B$ be the subsets of $\mathcal{P}(n)$ defined by

$$
\begin{gathered}
A=\left\{\lambda \in \mathcal{P}(n): F_{a, d}(\lambda)-F_{b, d}(\lambda)>\frac{(a+b) \sqrt{n}}{50 a b}\right\}, \\
B=\left\{\lambda \in \mathcal{P}(n): F_{a, d}(\lambda)-F_{b, d}(\lambda)<-\frac{(a+b) \sqrt{n}}{50 a b}\right\} .
\end{gathered}
$$

We know by Theorem 1.4 of [1] that $|A \cup B| \geqslant \frac{p(n)}{6}$. It remains to show that $|A| \geqslant|B|$. To do this, it is sufficient to prove that there exists a mapping $H: B \rightarrow A$ which is injective. This mapping will be defined by exchanging the parts $\equiv a(\bmod d)$ with parts $\equiv b(\bmod d)$.

(i) Case $b \neq 2 a$.

Since $0<a<b \leqslant d$, we have $b-a \not \equiv b(\bmod d)$ and $b-a \not \equiv a(\bmod d)$. (We supposed that $b \neq 2 a$, and we have $2 a-d<a<b$ ). To any $\lambda \in B$ with $\lambda: n=\sum_{j=1}^{n} j x_{j}$ we associate $H(\lambda): n=\sum_{j=1}^{n} j y_{j}$ with

$$
\begin{cases}y_{a+\ell d}=x_{b+\ell d} & \text { for } 0 \leqslant \ell<n / d \\ y_{b+\ell d}=x_{a+\ell d} & \text { for } 0 \leqslant \ell<n / d \\ y_{b-a}=x_{b-a}+F_{b, d}(\lambda)-F_{a, d}(\lambda) & \text { if } j \neq a, b(\bmod d) \text { and } j \neq b-a . \\ y_{j}=x_{j} & \end{cases}
$$

Note that $y_{b-a}>x_{b-a} \geqslant 0$ by $\lambda \in B$. It is clear that $H$ is injective because if $\lambda: n=\sum_{j=1}^{n} j x_{j}$ and $\lambda^{\prime}: n=\sum_{j=1}^{n} j x_{j}^{\prime}$ are such that $H(\lambda)=H\left(\lambda^{\prime}\right)$, then $\lambda$ and $\lambda^{\prime}$ are two partitions of $n$ with $x_{j}=x_{j}^{\prime}$ for all $j \neq b-a$. We check now that $H(\lambda)$ is a partition of $n$ :

$$
\begin{aligned}
\sum_{j=1}^{n} j y_{j} & =\sum_{\ell \geqslant 0}(a+\ell d) x_{b+\ell d}+\sum_{\ell \geqslant 0}(b+\ell d) x_{a+\ell d} \\
& +(b-a)\left(x_{b-a}+F_{b, d}(\lambda)-F_{a, d}(\lambda)\right)+\sum_{\substack{j \neq a, b(\bmod d) \\
j \neq b-a}} j x_{j} \\
= & n-(b-a) F_{b, d}(\lambda)+(b-a) F_{a, d}(\lambda)+(b-a)\left(F_{b, d}(\lambda)-F_{a, d}(\lambda)\right)=n .
\end{aligned}
$$

Finally we show that $H(\lambda) \in A$ :

$$
F_{a, d}(H(\lambda))-F_{b, d}(H(\lambda))=F_{b, d}(\lambda)-F_{a, d}(\lambda)>\frac{(a+b) \sqrt{n}}{50 a b} .
$$


(ii) Case $b=2 a$.

We take $H: B \rightarrow A$ defined by

$$
\begin{cases}y_{b+\ell d}=x_{a+\ell d} & \text { for } \ell \geqslant 0 \\ y_{a+\ell d}=x_{b+\ell d} & \text { for } \ell \geqslant 1 \\ y_{a}=F_{b, d}(\lambda)-F_{a, d}(\lambda)+x_{b} & \\ y_{j}=x_{j} & \text { for } j \neq \equiv a, b(\bmod d) .\end{cases}
$$

and we prove in the same way as in the previous case that this mapping is well defined and injective.

This completes the proof of Theorem 1.1.

\section{Regular partitions}

\subsection{On weakly regular partitions and proof of Theorem 1.2}

We will say that a partition $\lambda$ of $n$ is weakly regular if for all $0<a<a^{\prime} \leqslant d$ the inequality $F_{a, d}(\lambda) \geqslant F_{a^{\prime}, d}(\lambda)$ holds. Note that in the definition of $d$ regularity we have $>$ instead of $\geqslant$.

In this paragraph we will prove

Lemma 3.1. There are at least $\frac{p(n)}{d !}$ weakly $d$-regular partitions.

Proof. For $1 \leqslant j \leqslant d$, let $\mathcal{P}_{j}\left(a_{1}, \ldots, a_{j}\right)$ be the set of $\lambda \in \mathcal{P}(n)$ such that for all $1 \leqslant b \leqslant d$ with $b \notin\left\{a_{1}, \ldots, a_{j}\right\}$ we have

$$
F_{a_{1}, d}(\lambda) \geqslant \ldots \geqslant F_{a_{j}, d}(\lambda) \geqslant F_{b, d}(\lambda) .
$$

We will prove that for $1 \leqslant j \leqslant d$ (with $\mathcal{P}_{0}=\mathcal{P}(n)$ ) we have :

$$
\left|\mathcal{P}_{j}(1,2, \ldots, j)\right| \geqslant \frac{\left|\mathcal{P}_{j-1}(1,2, \ldots, j-1)\right|}{d-j+1} .
$$

Since

$$
\mathcal{P}_{j-1}(1,2, \ldots, j-1)=\cup_{j \leqslant a \leqslant d} \mathcal{P}_{j}(1,2, \ldots, j-1, a),
$$

it is sufficient to show that for all $j<a \leqslant d$ :

$$
\left|\mathcal{P}_{j}(1,2, \ldots, j-1, a)\right| \leqslant\left|\mathcal{P}_{j}(1,2, \ldots, j-1, j)\right| .
$$

Now we fix $j<a \leqslant d$. We define a mapping $F: \mathcal{P}_{j}(1,2, \ldots, j-1, a) \rightarrow$ $\mathcal{P}_{j}(1,2, \ldots, j-1, j)$ in the following way: to any $\lambda \in \mathcal{P}_{j}(1,2, \ldots, j-1, a)$, $\lambda: n=\sum_{k \geqslant 1} k x_{k}$ we associate $F(\lambda): n=\sum_{k \geqslant 1} k y_{k}$ with

$$
y_{k}= \begin{cases}x_{a+\ell d} & \text { if } k=j+\ell d \text { with } \ell \geqslant 0 \text { and } k \neq 1 \\ x_{j+\ell d} & \text { if } k=a+\ell d \text { with } \ell \geqslant 0 \\ x_{k} & \text { if } k \neq 1 \text { and } k \neq a, j(\bmod d),\end{cases}
$$


and

$$
y_{1}= \begin{cases}x_{1}+(a-j)\left(F_{a, d}(\lambda)-F_{j, d}(\lambda)\right) & \text { if } j \neq 1 \\ x_{a}+(a-1)\left(F_{a, d}(\lambda)-F_{1, d}(\lambda)\right) & \text { if } j=1 .\end{cases}
$$

So that the mapping $F$ exchanges the parts congruent to $\equiv a(\bmod d)$ with the parts congruent to $\equiv j(\bmod d)$, and then $y_{1}$ is chosen so that $F(\lambda)$ is a partition of $n$. In the same way as in the proof of Theorem 1.1 we check that $F$ is injective and $F(\lambda) \in \mathcal{P}_{j}(1, \ldots, j)$. This completes the proof of $(3 \cdot 1)$. Now we apply this inequality with $j=d-1, \ldots, 1$ :

$$
\left|\mathcal{P}_{d-1}(1,2, \ldots, d-1)\right| \geqslant \frac{\left|\mathcal{P}_{d-2}(1, d-1, \ldots, d-2)\right|}{2} \geqslant \ldots \geqslant \frac{\mathcal{P}(n)}{d !},
$$

and Lemma 3.1 is proved.

The $j=1$ special case of inequality $(3 \cdot 1)$ gives Theorem 1.2.

\subsection{Proof of Theorem 1.3}

Let $\overline{\mathcal{R}}(d)$ denote the set of the weakly $d$-regular partitions and $\mathcal{R}(d)$ the set of the $d$-regular partitions. By Lemma 3.1 it suffices to prove that

$$
|\mathcal{R}(d)|=|\overline{\mathcal{R}}(d)|+O\left(\frac{d^{4} p(n)}{\sqrt{n}}\right) .
$$

The basic idea of the proof of $(3 \cdot 2)$ is to assign to almost all $\lambda \in \overline{\mathcal{R}}(d)$ a partition $J(\lambda)$ such that for $2 \leqslant j \leqslant d$

$$
F_{j, d}(J(\lambda))=F_{j, d}(\lambda)-j-1 .
$$

This will assure $J(\lambda)$ to be regular. This mapping will be defined so that it replaces for each $2 \leqslant j \leqslant d, 1 \leqslant m \leqslant j-1$, a part equal to $j+m d$ by a convenient number of parts $\equiv 1(\bmod d)$. It is a direct consequence of the Proposition p. 159 of the paper of Erdős, Nicolas and Sárközy [3] that there is such a transformation for almost all partitions of $n$.

We recall their notation : if $\mathcal{A}=\left\{a_{1}, \ldots, a_{k}\right\}$ then $r(n, \mathcal{A})$ is the number of partitions of $n$ with no parts belonging to $\mathcal{A}$.

Proposition 3.2(Erdős, Nicolas and Sárközy [3] p. 159). There exists $c>0$ such that, if $\mathcal{A}=\left\{a_{1}, \ldots, a_{k}\right\}$ satisfies $u(\mathcal{A}):=a_{1}+\cdots+a_{k} \leqslant c n$ then when $n$ tends to infinity, we have

$$
\begin{aligned}
& r(n, \mathcal{A}) \leqslant\left(\prod_{i=1}^{k} a_{i}\right) p(n)\left(\frac{\pi}{\sqrt{6 n}}\right)^{k}\left(1+O\left(\frac{1}{\sqrt{n}}\right)\right), \\
& r(n, \mathcal{A}) \geqslant\left(\prod_{i=1}^{k} a_{i}\right) p(n)\left(\frac{\pi}{\sqrt{6 n}}\right)^{k} \exp \left(\frac{u(\mathcal{A})}{\sqrt{n}}\right) .
\end{aligned}
$$


We will use the upper bound $(3 \cdot 4)$ to obtain the following

Corollary 3.3. For almost all partitions of $n$ with at most $O\left(\frac{d^{4}}{\sqrt{n}} p(n)\right)$ exceptions, and for all $2 \leqslant j \leqslant d, 1 \leqslant m \leqslant j-1$, there is at least one part equal to $j+m d$.

Proof. Let $E$ be the number of partitions not having the property in Corollary 3.3. We have :

$$
\begin{aligned}
E & \leqslant \sum_{j=2}^{d} \sum_{m=1}^{j-1} r(n,\{j+m d\}) \\
& \ll \sum_{j=2}^{d} \sum_{m=1}^{j-1}(j+m d) \frac{p(n)}{\sqrt{n}} \\
& \ll p(n) \frac{d^{4}}{\sqrt{n}},
\end{aligned}
$$

by $(3 \cdot 4)$ which proves Corollary 3.3 .

Let $\overline{\mathcal{R}}^{+}(d)$ be the set of the partitions of $\overline{\mathcal{R}}(d)$ which have the property in Corollary 3.3. We have $\left|\overline{\mathcal{R}}^{+}(d)\right|=|\overline{\mathcal{R}}(d)|+O\left(\frac{d^{4} p(n)}{\sqrt{n}}\right)$. We define a mapping $J: \overline{\mathcal{R}}^{+}(d) \rightarrow \mathcal{R}(d)$ in the following way: for $\lambda: n=\sum_{k \geqslant 1} k x_{k}$, let $J(\lambda): n=\sum_{k \geqslant 1} k y_{k}$ with

$$
y_{k}=\left\{\begin{array}{lc}
x_{j+d m}-1 & \text { if } k=j+d m \text { with } 1 \leqslant m<j \leqslant d \\
x_{1+d m}+d-m & \text { if } k=1+d m \text { with } 1 \leqslant m \leqslant d-1 \\
x_{1}+(d-1)^{2}+\cdots+1^{2}=x_{1}+\frac{d(d-1)(2 d-1)}{6} \quad \text { if } k=1 & \text { otherwise. }
\end{array}\right.
$$

Note that since $\lambda \in \overline{\mathcal{R}}^{+}(d)$, for all $1 \leqslant m<j \leqslant d$, we have $x_{j+d m} \geqslant 1$ and $y_{j+d m} \geqslant 0$. First we show that we have $n=\sum_{k \geqslant 1} k y_{k}$. We put

$$
\mathcal{F}=\{1+m d: 0 \leqslant m \leqslant d-1\} \cup\{j+m d: 1 \leqslant m<j \leqslant d\} .
$$




$$
\begin{aligned}
\sum_{k \geqslant 1} k y_{k} & =\sum_{k \notin \mathcal{F}} k x_{k}+\sum_{j=2}^{d} \sum_{m=1}^{j-1}(j+d m)\left(x_{j+d m}-1\right) \\
& +\sum_{m=1}^{d-1}(1+d m)\left(x_{1+d m}+d-m\right)+x_{1}+\frac{d(d-1)(2 d-1)}{6} \\
& =n-\sum_{m=1}^{d-1} \sum_{j=m+1}^{d}(j+d m) \\
& +\sum_{m=1}^{d-1}(1+d m)(d-m)+\frac{d(d-1)(2 d-1)}{6}
\end{aligned}
$$

Then we remark that

$$
\begin{aligned}
\sum_{m=1}^{d-1} \sum_{j=m+1}^{d}(j+d m) & =\sum_{m=1}^{d-1} \sum_{j=m+1}^{d}(1+d m)+\sum_{m=1}^{d-1} \sum_{j=m+1}^{d}(j-1) \\
& =\sum_{m=1}^{d}(1+d m)(d-m)+\sum_{j=2}^{d}(j-1) \sum_{m=1}^{j-1} 1 \\
& =\sum_{m=1}^{d}(1+d m)(d-m)+\frac{d(d-1)(2 d-1)}{6}
\end{aligned}
$$

This proves that $\sum_{k \geqslant 1} k y_{k}=n$.

It is clear that condition (3.3) is satisfied and that $J$ is injective. Thus we have

$$
\begin{aligned}
|\mathcal{R}(d)| & \geqslant\left|\overline{\mathcal{R}}^{+}(d)\right| \\
& \geqslant|\overline{\mathcal{R}}(d)|+O\left(\frac{p(n) d^{4}}{\sqrt{n}}\right) \\
& \geqslant \frac{p(n)}{d !}\left(1+O\left(\frac{d ! d^{4}}{\sqrt{n}}\right)\right),
\end{aligned}
$$

This ends the proof of Theorem 1.3.

\section{Parts in residue classes of large moduli}

In this paragraph we prove Proposition 1.5.

When $d$ is large we will use a result of Szalay and Turán [5]. Recall that at the beginning of the introduction we defined $\lambda_{1}$ as the greatest part of the partition $\lambda$. 
Lemma 4.1(Szalay and Turán [5] Lemma 4 p.137). The inequality

$$
\lambda_{1} \leqslant \frac{5 \sqrt{6}}{2 \pi} \sqrt{n} \log n
$$

holds with the exception of at most $0\left(p(n) n^{-2}\right)$ partitions $\lambda$.

By this Lemma it is clear that for almost all partitions with at most $0\left(p(n) n^{-2}\right)$ exceptions we have

$$
F_{0, d}(\lambda)=0 \text { for all } d>\frac{5 \sqrt{6}}{2 \pi} \sqrt{n} \log n .
$$

Now we suppose that

$$
d \leqslant \frac{5 \sqrt{6}}{2 \pi} \sqrt{n} \log n
$$

Consider a partition $\lambda$ satisfying (1.3), and let $\lambda_{i_{1}}=d \mu_{1}, \ldots, \lambda_{i_{t}}=d \mu_{t}$ denote the parts divisible by $d$ (so that $\mathrm{t}=F_{0, d}(\lambda)$ ), and write

$$
\mu_{1}+\cdots+\mu_{t}=u
$$

Then $\mu=\left(\mu_{1}, \ldots, \mu_{t}\right)$ is a partition of $u$ with

$$
t=F_{0, d}(\lambda) \geqslant 100 \frac{\sqrt{n} \log n \log ^{2} d}{d}=: Z
$$

by $(1 \cdot 3)$, and the $\lambda_{j}$ 's different from $\lambda_{i_{1}}, \ldots, \lambda_{i t}$ form a partition of $n-d u$. Thus the number of partitions $\lambda$ of $n$ satisfying (1.3) is

$$
\leqslant \sum_{u \leqslant n / d} H(u) p(n-u d)
$$

where $H(u)$ is the number of partitions of $u$ into at least $Z$ parts, which is the same as the number of partitions of $u$ with the property that the greatest part $\lambda_{1}$ is $\geqslant Z$.

When $u$ is "small", $u \leqslant U$ with

$$
U:=\frac{100 n \log ^{2} d}{d^{2}}
$$

we will use an upper bound of $H(u)$ obtained by Szalay and Turán [5] and when $u>U$ is "large", $p(n-d u)$ will be small and it will be sufficient to take $H(u) \leqslant p(u)$. 
Lemma 4.2(Szalay and Turán [5] Lemma 3 p. 136). If $\beta=\beta(n)$ is restricted by

$$
0<\beta<\frac{\pi}{2 \sqrt{6}} \frac{\sqrt{n}}{\log n}-\frac{1}{2}
$$

then the relation

$$
\lambda_{1} \leqslant(1+2 \beta) \frac{\sqrt{6}}{2 \pi} \sqrt{n} \log n
$$

holds with the exception of $O\left(p(n) n^{-\beta}\right) \lambda$ 's at most.

Remark: Lemma 4.1 is the $\beta=2$ special case of Lemma 4.2.

We start with the splitting

$$
\begin{aligned}
\sum_{u \leqslant n / d} H(u) p(n-u d) & =\sum_{Z \leqslant u \leqslant U} H(u) p(n-u d)+\sum_{U<u \leqslant n / d} H(u) p(n-u d) \\
& =S_{1}+S_{2} .
\end{aligned}
$$

For $S_{2}$ we use the upper bound $H(u) \leqslant p(u)$ and then we apply the well known estimation of Hardy and Ramanujan [4]

$$
p(n)=\frac{1}{4 n \sqrt{3}} e^{C \sqrt{n}}\left(1+O\left(n^{-1 / 2}\right)\right)
$$

Thus we have

$$
S_{2} \ll \sum_{U<u \leqslant n / d} \frac{\exp (C \sqrt{u}+C \sqrt{n-d u})}{u(n-d u)} .
$$

Now we use the elementary facts that $\frac{1}{u(n-u d)}=\frac{1}{n u}+\frac{d}{n(n-u d)}$ and $\frac{1}{u} \leqslant \frac{d}{n-u d}$ if and only if $u \geqslant \frac{n}{2 d}$. This yields to the upper bound

$$
\begin{aligned}
S_{2} & \ll \sum_{U<u \leqslant \frac{u}{2 d}} p(n) \frac{\exp (C \sqrt{u}+C \sqrt{n-u d}-C \sqrt{n})}{u} \\
& +p(n) \sum_{\frac{u}{2 d}<u \leqslant \frac{n}{d}} \frac{d \exp (C \sqrt{u}+C \sqrt{n-u d}-C \sqrt{n})}{n-u d} .
\end{aligned}
$$


The function $u \rightarrow \sqrt{u}+\sqrt{n-u d}$ is decreasing on the interval $\left[n /\left(d^{2}+\right.\right.$ $d), n / d]$ and by $(4 \cdot 5) U>n /\left(d^{2}+d\right)$ :

$$
\begin{aligned}
S_{2} & \ll p(n) \log \left(\frac{n}{2 d U}\right) \exp (C \sqrt{U}+C \sqrt{n-U d}-C \sqrt{n}) \\
& +p(n) d \log n \exp \left(C \sqrt{\frac{n}{2 d}}+C \sqrt{\frac{n}{2}}-C \sqrt{n}\right) .
\end{aligned}
$$

When $d \geqslant 8$, the second term in the right member of (4.9) is less than

$$
p(n) d \log n \exp (-0.04 C \sqrt{n}) \ll p(n) d^{-2} .
$$

Using the inequality $\sqrt{1-t} \leqslant 1-t / 2$ for $t \in[0,1]$ and the definition of $U$ in $(4 \cdot 5)$ the first term is

$$
\begin{aligned}
& \ll p(n)(\log d) \exp \left(10 C \frac{\log d}{d} \sqrt{n}-\frac{50 C \log ^{2} d \sqrt{n}}{d^{2}}\right) \\
& \ll p(n)(\log d) \exp \left(-40 C \frac{\log ^{2} d \sqrt{n}}{d}\right) \\
& \ll p(n)(\log d) \exp \left(-\frac{32 \pi^{2}}{9} \log d\right) \\
& \ll p(n) d^{-2}
\end{aligned}
$$

when $8 \leqslant d \leqslant 5 \frac{\sqrt{6}}{2 \pi} \sqrt{n} \log n$. Thus we have

$$
S_{2} \ll p(n) d^{-2} .
$$

Now we will use Lemma 4.2 to obtain an upper bound for $H(u)$ in $S_{1}$. We will take

$$
\beta=\beta(u)=\frac{\pi Z}{\sqrt{6 u} \log u}-\frac{1}{2} .
$$

The inequalities in (4.6) are satisfied if we have

$$
u \log ^{2} u<\frac{2 \pi^{2} Z^{2}}{3} \text { and } u>2 Z .
$$

With our choices of $Z$ and $U,(4 \cdot 3)$ and (4.5), we see that the first inequality is satisfied when $u \leqslant U$. The inequality $\beta<\frac{\pi}{2 \sqrt{6}} \frac{\sqrt{u}}{\log u}-\frac{1}{2}$ in (4.6) is verified 
if $u>2 Z$. For $Z \leqslant u \leqslant 2 Z$ we will replace $H(u)$ by $\tilde{H}(u)$ the number of partitions of $u$ with at least $Z / 4$ parts:

$$
S_{1} \leqslant \sum_{Z \leqslant u \leqslant 2 Z} \tilde{H}(u) p(n-u d)+\sum_{2 Z<u \leqslant U} H(u) p(n-u d) .
$$

By Lemma 4.2 we have

$$
\begin{aligned}
& H(u) \ll p(u) u^{-\beta(u)} \text { for } 2 Z<u \leqslant U \\
& \tilde{H}(u) \ll p(u) u^{-\tilde{\beta}(u)} \text { for } Z \leqslant u \leqslant 2 Z
\end{aligned},
$$

with $\tilde{\beta}(u)=\frac{\pi Z}{4 \sqrt{6 u} \log u}-\frac{1}{2}$. With these upper bounds, we obtain for $(4 \cdot 12)$

$$
\begin{aligned}
S_{1} & \ll \sum_{Z \leqslant u \leqslant 2 Z} p(u) p(n-u d) \exp \left(-\left(\frac{\pi \sqrt{Z}}{4 \sqrt{6 u} \log u}-\frac{1}{2}\right) \log u\right) \\
& +\sum_{2 Z \leqslant u \leqslant U} p(u) p(n-u d) \exp \left(-\left(\frac{\pi \sqrt{Z}}{\sqrt{6 u} \log u}-\frac{1}{2}\right) \log u\right) \\
& \ll p(n)\left(\sqrt{Z} \exp \left(\frac{-\pi \sqrt{Z}}{8 \sqrt{3}}\right)+\sqrt{U} \exp \left(-\frac{\pi Z}{\sqrt{6 U}}\right)\right) .
\end{aligned}
$$

When $8 \leqslant d \leqslant \frac{5 \sqrt{6}}{2 \pi} \sqrt{n} \log n$, we have

$$
\begin{aligned}
\sqrt{Z} \exp \left(-\frac{\pi \sqrt{Z}}{8 \sqrt{3}}\right) & \leqslant 10 \frac{\sqrt{2 \pi}}{\sqrt{5 \sqrt{6}}} \log \left(\frac{5 \sqrt{6}}{2 \pi} \sqrt{n} \log n\right) \exp \left(-\frac{5 \pi}{4 \sqrt{3}} \sqrt{\frac{2 \pi}{5 \sqrt{6}}} \log n\right) \\
& \ll n^{-2}
\end{aligned}
$$

By $(4 \cdot 5)$ and $(4 \cdot 3)$ we also have

$$
\left.\sqrt{U} \exp \left(-\frac{\pi Z}{\sqrt{6 U}}\right)\right) \leqslant \frac{10 n}{d \log d} \exp \left(-\frac{10 \pi}{\sqrt{6}} \log n \log d\right)
$$

We have proved that $S_{1} \leqslant p(n) n^{-2}$, which completes the proof of Proposition 1.5. 


\section{The number of the square free parts ; proof of the Theorem 1.4}

Let $N(\lambda)$ denote the number of the square free parts in the partition $\lambda$ and recall that $s(\lambda)$ is the number of parts of $\lambda$. Erdős and Lehner [2] proved that for almost all $\lambda, s(\lambda)=\frac{\sqrt{n} \log n}{C}(1+o(1))$. Let $K$ be arbitrarily large but fixed. Let $M:=\prod_{p \leqslant K} p^{2}$ and

$$
\mathcal{M}:=\left\{1 \leqslant a \leqslant M: a \not \equiv 0\left(\bmod p^{2}\right) \text { for all } p<K\right\} .
$$

It follows from Theorem 1.3 in [1] that for $a \in \mathcal{M}$ and for almost all partitions we have

$$
F_{a, M}(\lambda)=\frac{\sqrt{n} \log n}{M C}(1+o(1)) .
$$

Thus for almost all partitions the number $N_{1}(\lambda)$ of parts without a divisor $p^{2}$ with $p \leqslant K$ is

$$
\begin{aligned}
N_{1}(\lambda) & =\frac{\sqrt{n} \log n}{M C}|\mathcal{M}|(1+o(1)) \\
& =\prod_{p \leqslant K}\left(1-\frac{1}{p^{2}}\right) \frac{\sqrt{n} \log n}{C}(1+o(1)) \\
& =\frac{6}{\pi^{2}}\left(1+O\left(K^{-1}\right)\right) \frac{\sqrt{n} \log n}{C}(1+o(1)) .
\end{aligned}
$$

It remains to show that for more than $(1-\varepsilon) p(n)$ partitions the number $N_{1}(\lambda)-N(\lambda)$ of parts divisible by some $p^{2}$ with $p>K$ is small enough. This will be done by using Proposition 1.5 :

$$
\begin{aligned}
N_{1}(\lambda)-N(\lambda) & \leqslant \sum_{p>K} F_{0, p^{2}}(\lambda) \\
& \leqslant \sum_{p>K} 100 \frac{\sqrt{n} \log n\left(\log p^{2}\right)^{2}}{p^{2}} \ll \frac{\sqrt{n} \log n \log K}{K}
\end{aligned}
$$

for almost all partitions with at most $\sum_{p>K} \frac{p(n)}{p^{4}} \ll p(n) K^{-3}$ exceptions. 


\section{References}

[1] C. Dartyge and A. Sárközy, Arithmetic properties of summands of partitions to appear in Ramanujan Journal.

[2] P. Erdös and J. Lehner, The distribution of the number of summands in the partitions of a positive integer, Duke Math. Journal 8 (1941), 335-345.

[3] P. Erdős, J.-L Nicolas, and A. Sárközy, On the number of partitions of $n$ without a given subsum (I), Discrete Math. 75 (1989), 155-166.

[4] G. H. Hardy and S. Ramanujan, Asymptotic formulae in combinatory analysis, Proceedings of the London Math. Soc. vol. 17 (1918), 465-490.

[5] M. Szalay and P. Turán, On some problems of a statistical theory of partitions with application to character of the symmetric group III, Acta Math. Acad. Sci. Hungar. 32 (1978), 129-155.

\section{Cécile Dartyge}

Institut Élie Cartan

Université Henri Poincaré-Nancy 1

BP 239

54506 Vandœuvre Cedex

France

dartyge@iecn.u-nancy.fr

\author{
András Sárközy \\ Department of Algebra and Number Theory \\ Eötvös Loránd University \\ H-1518 Budapest, Pf. 120 \\ 1117 Budapest, Pázmány Péter Sétány 1/C \\ Hungary \\ sarkozy@cs.elte.hu
}

